\title{
Les politiques de développement à l'épreuve de la territorialisation
}

Changements et stabilités dans une situation de décolonisation négociée, la province nord de la Nouvelle-Calédonie

\section{Séverine Bouard}

\section{(2) OpenEdition}

\section{Journals}

Édition électronique

URL : http://journals.openedition.org/cdg/1029

DOI : $10.4000 /$ cdg. 1029

ISSN : 2107-7266

Éditeur

UMR 245 - CESSMA

Référence électronique

Séverine Bouard, «Les politiques de développement à l'épreuve de la territorialisation », Carnets de géographes [En ligne], 4 | 2012, mis en ligne le 01 septembre 2012, consulté le 24 septembre 2020. URL : http://journals.openedition.org/cdg/1029 ; DOI : https://doi.org/10.4000/cdg.1029

\section{(c) $(1) \odot$}

La revue Carnets de géographes est mise à disposition selon les termes de la Licence Creative Commons Attribution - Pas d'Utilisation Commerciale - Pas de Modification 4.0 International. 


\title{
LES POLITIQUES DE DEVELOPPEMENT A L'EPREUVE DE LA TERRITORIALISATION
}

\author{
Changements et stabilités dans une situation de décolonisation \\ négociée, la province nord de la Nouvelle-Calédonie
}

Séverine BOUARD

Les dispositifs de développement territorial multiplient les territoires de gouvernance et les acteurs qui interviennent dans la fabrication de l'action publique. Cela pose la question théorique des modalités d'interactions de ces acteurs dans leur diversité, leurs échelles et périmètres d'intervention et la question des effets de ces interactions sur le processus de fabrication de l'action publique.

En associant géographie et science politique, la thèse propose un modèle d'analyse d'inspiration foucaldienne pour répondre à cette question. Dans cette recherche, nous nous penchons plus spécifiquement sur la dimension politique et institutionnelle du territoire (LEVY, 1994). Le territoire n'est donc pas forcément synonyme de local, mais plutôt un espace géographique et social institutionnalisé faisant l'objet d'une légitimation permanente. Dans cette perspective, tout territoire est caractérisé par des répertoires cognitifs et symboliques utilisés par les acteurs pour étayer leur discours et/ou favoriser la formation d'alliances avec d'autres acteurs afin de légitimer leur position dans l'espace géographique et social institutionnalisé.

Pour chaque niveau de territoire considéré, nous proposons d'analyser " l'étoile " de l'action publique, expression empruntée à P. LASCOUMES et P. LE GALES (2009), qui articule les différentes variables qui influencent les processus de changement des politiques publiques: les représentations, les acteurs, les instruments de politiques publiques, les institutions et les résultats des politiques publiques. Ce cadre est utilisé pour expliquer le processus observé dans la province Nord de Nouvelle-Calédonie.

Le territoire de la province Nord est original à deux titres pour traiter cette question. D'une part, l'histoire de la Nouvelle-Calédonie est marquée par un dualisme entre un modèle de développement " européen " professionnel et marchand, et un modèle de développement "kanak" multifonctionnel et non marchand (SOURISSEAU, PESTANA et al. 2010). Issu de la colonisation et de la mise en réserve des Kanak (MERLE 1995, 
DAUPHINE 1989) le dualisme semble se maintenir malgré une large réattribution du foncier aux Kanak dans le cadre de la réforme foncière. D'autre part, depuis la signature des Accords de Matignon (1989), la Nouvelle-Calédonie est divisée en trois provinces dont la province Nord, gérée par des élus kanak majoritairement indépendantistes. Ainsi, après avoir été pratiquement exclus du processus d'élaboration des politiques publiques (SORIANO 2000, TREPIED 2007), les nouveaux élus kanak peuvent mettre en œuvre les politiques publiques de développement qu'ils entendent. Après la signature de l'Accord de Nouméa (1998) qui inscrit la NouvelleCalédonie dans un processus de décolonisation garantissant une autonomie croissante, un dispositif de développement territorial, venant de la France, a été mis en œuvre sur les communes du territoire provincial: les Opérations groupées d'aménagement du foncier (OGAF). Le dispositif s'appuie sur une association locale composée d'acteurs du territoire de projet et sur des comités locaux de discussion qui favorisent la participation d'un plus grand nombre d'acteurs aux politiques de développement. Après quelques expérimentations, les OGAF sont devenues centrales dans la politique provinciale de développement.

Les résultats reposent sur 64 entretiens semi-directifs à but informatif et compréhensif: entretiens auprès des élus provinciaux et des chargés de mission, auprès des services de l'Etat français, d'experts et de chercheurs. L'analyse de ces entretiens a été complétée par la collecte d'une documentation grise hétérogène (déclaration de politiques publiques, textes de loi, réglementation, diagnostics, évaluation et travaux d'expertise, échanges de courrier et comptes rendus de réunions, bases de données) et la réalisation d'observations ethnographiques.

En analysant les discours et les pratiques des acteurs, la thèse examine les interactions multi-niveaux qui se nouent entre les acteurs autour des usages de l'instrument OGAF. Cette thèse souligne que le dispositif est mobilisé de manières diversifiées par les acteurs selon leurs trajectoires, leurs représentations du développement et leurs intérêts.

Au niveau provincial, les interactions entre experts et élus provinciaux permettant le transfert de l'instrument OGAF montrent la construction d'alliances traductionnelles et une instrumentalisation réciproque.

Les usages locaux du dispositif OGAF soulignent que l'entrée de nouveaux acteurs dans la mise en œuvre est mesurée. Les stratégies de recrutements des membres des comités locaux se révèlent proches des stratégies de recrutements des partis politiques.

Notre recherche s'intéresse ensuite aux effets de l'ouverture des comités locaux en caractérisant les recompositions dans les discours et les pratiques des acteurs en termes de développement. La thèse démontre la constitution d'une coalition discursive ascendante vers le niveau provincial permettant de légitimer l'institutionnalisation des OGAF au sein des politiques provinciales. 
Enfin, l'analyse des OGAF récentes montre que les élus provinciaux et les cadres de l'administration provinciale utilisent l'instrument pour mieux contrôler la partie Est du territoire provincial.

\section{Références:}

DAUPHINE, J. (1989). Les spoliations foncières en Nouvelle-Calédonie. Paris, L'Harmattan

LASCOUMES, P., et LE GALES, P., (2009) Sociologie de I'action publique, Armand Colin, Paris

LEVY, J., (1994) L'espace légitime: sur la dimension géographique de la fonction politique, Paris, Presses de la Fondation nationale des sciences politiques,.

MERLE, I. (1995). Expériences coloniales : la Nouvelle-Calédonie. 1853-1920. Paris, Belin.

SOURISSEAU, J.M., GAILLARD, C., PESTANA, G., BOUARD, S., MENNESSON, T., (2010). A la recherche des politiques publiques agricoles et rurales. Trajectoires des institutions et représentations locales des enjeux de développement. Nouméa, IAC Editions, coll. Etudes et synthèses.

SORIANO, E. (2000). Une trajectoire du politique en Mélanésie. Construction identitaire et émergence d'un personnel politique. L'exemple Kanak de Nouvelle-Calédonie (19461999). Science politique. Montpellier, Montpellier I. Thèse de doctorat.

TREPIED, B. (2007). Politique et relations coloniales en Nouvelle-Calédonie. Ethnographie historique de la commune de Koné, 1946 -1988. Anthropologie. Paris, EHESS. Thèse de doctorat. 


\section{Fiche informative}

\section{Lien électronique vers la thèse}

http://tel.archives-ouvertes.fr/docs/00/65/64/17/PDF/these finale sb decembre 2011.pdf

\section{Discipline}

Géographie

\section{Directeur}

Jean-Philippe TONNEAU, directeur de l'UMR Tetis, CIRAD.

\section{Université}

Université Paul VALERY Montpellier III

Membres du jury de thèse, soutenue le 19 octobre 2011

- M. GUMUCHIAN, Professeur émérite des Universités, Université de Grenoble I, rapporteur et président du jury.

- M. LE MEUR Pierre-Yves, Chercheur Habilité, IRD, rapporteur.

- M. JOBERT Bruno, Directeur de recherche, Professeur émérite des Universités, Université de Grenoble, CNRS.

- M. CHEVALIER Pascal, Maître de conférences, Université de Montpellier III.

- M. SOURISSEAU Jean-Michel, Chercheur, CIRAD.

\section{Courriel de l'auteur}

severinebouard@hotmail.fr 